Col I i si onal - radi at i ve model i ncl udi ng reconbi nat i on processes for WR7+ i on

\begin{tabular}{|l|l|}
\hline $\begin{array}{l}\text { jour nal or } \\
\text { publ i cat i on ti t e }\end{array}$ & $\begin{array}{l}\text { The Eur opean Physi cal Journal D, At omi c, } \\
\text { nol ecul ar and opt i cal physi cs }\end{array}$ \\
\hline vol une & 71 \\
\hline page range & 246 \\
\hline year & $2017-10-10$ \\
\hline URL & ht t p: //hdl . handl e. net /10655/00012841 \\
\hline
\end{tabular}




\section{Collisional-radiative model including recombination processes for}

\section{$\mathbf{W}^{27+}$ ion}

Izumi Murakami ${ }^{1,2}$, Akira Sasaki ${ }^{3}$, Daiji Kato ${ }^{1,2}$ and Fumihiro Koike ${ }^{4}$

${ }^{1}$ National Institute for Fusion Science, National Institutes of Natural Sciences, Toki, Gifu 509-5292, Japan

${ }^{2}$ Department of Fusion Science, SOKENDAI (The Graduate University of Advanced Studies), Toki, Gifu 509-5292, Japan

${ }^{3}$ Kansai Photon Science Institute, National Institutes of Quantum and Radiological Science and Technology, Kizugawa, Kyoto 619-0215, Japan

4 Sophia University, Chiyoda-ku, Tokyo 102-8554, Japan

Received: date / Revised version: date

Abstract. We have constructed a collisional-radiative $(\mathrm{CR})$ model for $\mathrm{W}^{27+}$ ions including 226 configurations with $n \leq 9$ and $l \leq 5$ for spectroscopic diagnostics. We newly include recombination processes in the model and this is the first result of extreme ultraviolet spectrum calculated for recombining plasma component. Calculated spectra in 40-70 Å range in ionizing and recombining plasma components show similar 3 strong lines and 1 weak line in recombining plamsa component at 45-50 $\AA$ and many weak lines at 50-65 $\AA$ for both components. Recombination processes do not contribute much to the spectrum at around $60 \AA$ for $\mathrm{W}^{27+}$ ion. Dielectronic satellite lines are also minor contribution to the spectrum of recombining plasma component. Dielectronic recombination (DR) rate coefficient from $\mathrm{W}^{28+}$ to $\mathrm{W}^{27+}$ ions is also calculated with the same atomic data in the CR model. We found that larger set of energy levels including many autoionizing states gaves larger DR rate coefficients but our rate agree within factor 6 with other works at electron temperature around $1 \mathrm{keV}$ in which $\mathrm{W}^{27+}$ and $\mathrm{W}^{28+}$ ions are usually observed in plasmas.

PACS. 32.30.-r Atomic spectra

\section{Introduction}

Tungsten is used as a plasma facing material in fusion devices such as ASDEX Upgrade, JET and ITER. Tung- sten impurity in the main plasma is of great concern to transport study in plasma, since large radiation power of partially ionized tungsten reduces plasma temperature 
and affects fusion performance. We require a spectroscopic model for tungsten ions in order to study tungsten behavior in plasma and to estimate the radiation power loss from tungsten spectra.

Extreme ultraviolet (EUV) spectra of tungsten ions measured in plasma with electron temperature around 1$2 \mathrm{keV}$ in various fusion devices, such as ASDEX Upgrade and Large Helical Device (LHD), show a characteristic feature at 45 - $70 \AA$, the so-called unresolved transition array (UTA) [1], [2]. Overlapped numerous emission lines produce a wide two-peak continuum-like structure. This structure is produced by the principal quantum number $n=4-4$ transitions of $\mathrm{W}^{q+}$ with $\mathrm{q} \sim 25-34$ [3]. This UTA structure is not seen for plasma with electron temperature higher than $3 \mathrm{keV}$ [4].

Pütterich et al. [5] and Murakami et al. [3] attempted to reproduce the UTA feature with their own collisionalradiative (CR) models, but only one peak at $\sim 50 \AA$ was produced and the second peak at $\sim 60 \AA$ was not produced. Pütterich et al. suggested that the dielecronic recombination (DR) process might contribute to this spectral feature, but none of the CR models included recombination processes in the models.

There are some theoretical works reported on DR rate coefficients for tungsten ions (e.g., [6], [7] and references therein). Total DR recombination rate coefficients are given as a function of electron temperature in those works. Such total rates can be used in calculations of ionization balance of ion densities and transport study. In a CR model, state-selective rate coefficients are necessary for all ex- cited states. However, energy levels calculated by different atomic codes do not agree well for excited states with higher $n$ and it is very difficult to make matching levels between different sets of energy levels. Therefore, even if the previous works give state-selective recombination rate coefficients, they would not be used in a CR model constructed with different atomic data sets.

Sasaki and Murakami [8] constructed a CR model with large numbers of configuration averaged levels including autoionizing levels (hereafter refered to as CA-CR model). They used a computer algorithm to make a large set of electron configurations by determining core configurations [9]. Inclusion of a large number of autoionizing levels allowed treatment of dielectronic capture and autoionization processes explicitly, and the effect of the DR process could be included in the CA-CR model.

Using this CA-CR model, the effective recombination rate coefficients were calculated for $\mathrm{W}^{27+}$ and $\mathrm{W}^{26+}$ ions by Murakami and Sasaki [10]. Because many dielectronic capture channels were included, obtained rate coefficients at $100 \mathrm{eV}-1 \mathrm{keV}$ were much larger than those obtained by Safronova et al. [11] and $\mathrm{Li}$ et al. [12], using an ordinal method, i.e., including a limited number of autoionizing states in the calculation, and adding contribution from high $n$ levels using n-scaling law. Although Murakami and Sasaki did not include high $n$ contribution to the rate coefficients, the rate becomes larger than the ordinal calculations. This CA-CR model has the advantage of obtaining better recombination rates, but is not suitable for calcu- 
lating spectra because of the configuration averaged levels used.

For spectroscopic diagnostics, we require a CR model with J-resolved fine structure levels. We plan to extend the CR model for tungsten ions of Murakami et al.[3] to include recombination processes to examine the effect of recombination processes in tungsten EUV spectra. This model was constructed with an immense number of Jresolved fine structure levels in which autoionizing levels were not yet included. As a first step, we focus on $\mathrm{W}^{27+}$ ion here to examine the effect of recombination processes in the EUV spectra. The ground state configuration of this ion is simple as $4 \mathrm{~d}^{10} 4 \mathrm{f}$, and it is easy to extend to include autoionizing levels in the CR model. Thus we construct an extended CR model with J-resolved fine structure levels with the principal quantum number $n$ up to 9 and autoionizing levels. We include dielectronic capture and autoionization instead of including DR process. The radiative recombination and three-body recombination processes are included in the CR model. With this model we calculate EUV spectra of $\mathrm{W}^{27+}$ ions for ionizing and recombining plasma.

In the following, we describe the details of our CR model in section 2, and show calculated EUV spectra in section 3. In section 4 , DR rate coefficients and satellite lines are shown. Summary is given in section 5 .

\section{Collisional-Radiative Model}

A CR model is for calculating population densities of excited states for an ion with assumption of quasi-steady- state. Here we construct the $\mathrm{CR}$ model for $\mathrm{W}^{27+}$ ion. Population densities of the excited states are determined by radiative processes and collisional processes between excited states. Usually radiative decay, electron-impact ionization, and electron-impact excitation and de-excitation processes are considered in the model as found in $\mathrm{Mu}-$ rakami et al.[3]. Here we also include radiative recombination and three-body recombination from the ground state of the $\mathrm{W}^{28+}$ ion. In addition, we also examine population densities of autoionizing states. Autoionization and dielectronic capture processes for the autoionizing states of $\mathrm{W}^{27+}$ ion are considered as well as with other collisional and radiative processes. These processes are necessary to include the effect of DR process. We examine all of these processes for J-resolved fine structure levels of $\mathrm{W}^{27+}$ ion.

The rate equation for the population density $n(i)$ of the excited level $i$ is described as

$$
\begin{aligned}
d n(i) / d t= & -\left(\sum_{k>i} C(i, k) n_{e}\right. \\
& +\sum_{j<i}\left(F(i, j) n_{e}+A_{r}(i, j)\right) \\
& \left.+S(i) n_{e}+\sum_{i_{0}^{\prime}} A_{a}\left(i, i_{0}^{\prime}\right)\right) n(i) \\
& +\sum_{j<i} C(j, i) n_{e} n(j) \\
& +\sum_{k>i}\left(F(k, i) n_{e}+A_{r}(k, i)\right) n(k) \\
& +\left(\beta(i) n_{e}^{2}+\alpha_{r}(i) n_{e}+D_{c}(i) n_{e}\right) n_{0}
\end{aligned}
$$

where $j$ denotes a level lower than the level $i$ including the ground state, $k$ denotes an excited level higher than the level $i, C(i, j)$ and $F(i, j)$ are electron-impact excitation and de-excitation rate coefficients, $A_{r}(i, j)$ is radiative transition rate from $i$ to $j$ levels, $S(i)$ is electron-impact 
ionization rate coefficient, $A_{a}\left(i, i_{0}^{\prime}\right)$ is autoionization rate from $i$ to the ground state or excited state $i_{0}^{\prime}$ of $\mathrm{W}^{28+}$ ion, $\beta(i)$ is three-body recombination rate coefficient, $\alpha_{r}(i)$ is radiative recombination rate coefficient, $D_{c}(i)$ is dielectronic capture rate coefficient, $n_{e}$ is electron density, and $n_{0}$ is the ground state density of $\mathrm{W}^{28+}$ ion.

A set of the rate equations are solved with steady-state assumption, where electron temperature and density are given. The population density is obtained as a combination of an ionizing and a recombining plasma components. These are proportional to the population densities of the ground state of $\mathrm{W}^{27+}$ ion and the ionized state, i.e., the ground state of $\mathrm{W}^{28+}$ ion, respectively.

$$
n(i)=N_{1}(i) n_{1}+N_{0}(i) n_{0}
$$

where $N_{1}(i)$ and $N_{0}(i)$ are an ionizing and a recombining plasma population coefficients, respectively. The ion density ratio, $n_{1} / n_{0}$ should be determined by another method.

Atomic data of energy levels, radiative transition rates, electron-impact ionization and excitation cross sections, autoionization rates, and photoionization cross sections are calculated with the HULLAC atomic code (ver.9) [13]. The rate coefficients are calculated from the cross sections with Maxwellian electron velocity distribution. Electronimpact de-excitation, radiative recombination, three-body recombination, and dielectronic capture rate coefficients are calculated from the rate coefficients of their inverse processes under the detailed balance condition.

In this work, we consider 226 electron configurations of $\mathrm{W}^{27+}$ ions as $4 \mathrm{~d}^{10} 4 \mathrm{f}, 4 \mathrm{~d}^{10} n l(n=5-9, l=0-5), 4 \mathrm{~d}^{9} 4 \mathrm{f}^{2}$, $4 \mathrm{~d}^{9} 4 \mathrm{f} n l(n=5-9, l=0-5), 4 \mathrm{~d}^{9} 5 \ln l^{\prime}\left(n=5-8, l, l^{\prime}=0-\quad\right.$ level.

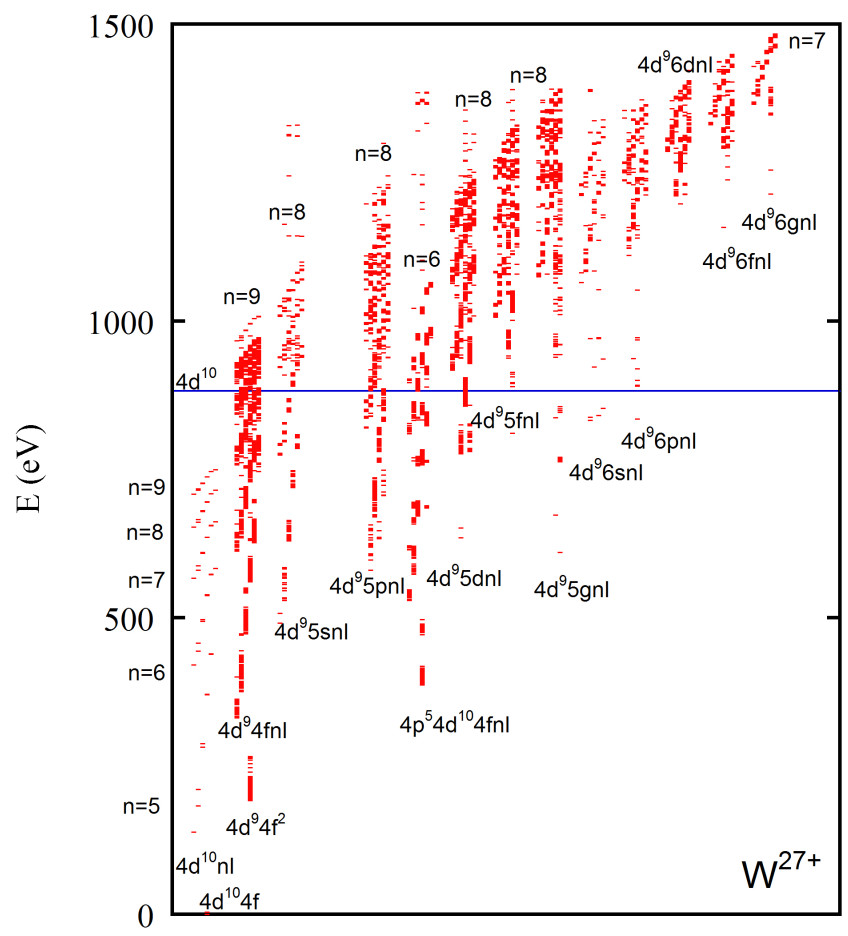

Fig. 1. Energy level diagram for $\mathrm{W}^{27+}$ ions. Ionization threshold at $\mathrm{E}=873 \mathrm{eV}$ is indicated as a horizontal line.

$5), 4 \mathrm{~d}^{9} 6 \ln l^{\prime}\left(n=6-7, l=0-4, l^{\prime}=0-5\right), 4 \mathrm{p}^{5} 4 \mathrm{~d}^{10} 4 \mathrm{f}^{2}$, and $4 \mathrm{p}^{5} 4 \mathrm{~d}^{10} 4 \mathrm{f} n l(n=5-6, l=0-5)$, and 25,632 J-resolved levels are included in the model. Configuration interactions are taken into account when atomic structure is calculated with the HULLAC code. We treat a 25,631 × 25,631 matrix of coefficients to solve the rate equation (1).

Figure 1 shows the energy level diagram of $\mathrm{W}^{27+}$ ion. Only 4,325 levels are below the ionization threshold, i.e., the ground state $4 \mathrm{~d}^{10}$ of $\mathrm{W}^{28+}$ ion, and other 21,287 levels are autoionizing levels. Since levels with $n$ up to 9 are considered for the excited states $4 \mathrm{~d}^{10} n l$, there is a gap between the ionization threshold and the highest $4 \mathrm{~d}^{10} 9 l$ 
Emission line intensity for the transition from level $i$

to level $j$ is obtained as

$$
I(i, j)=n(i) A_{r}(i, j) \Delta E(i, j)
$$

where $\Delta E(i, j)$ is the transition energy.

\section{EUV spectra}

EUV spectra of $\mathrm{W}^{27+}$ ion for both ionizing and recombining plasmas are obtained by solving the rate equations with given electron temperature and density. Figure 2 shows the EUV spectra for both plasmas with assuming the electron temperature as $1 \mathrm{keV}$ and the electron density as $10^{13} \mathrm{~cm}^{-3}$. The intensities are normalized with the ion densities of $\mathrm{W}^{27+}$ and $\mathrm{W}^{28+}$ ions for ionizing and recombining plasmas, respectively. Spectra are convolved with a Gaussian profile with resolution $\Delta \lambda / \lambda=0.002$. For comparison, a typical experimental tungsten UTA spectrum measured in LHD plasma is shown in Fig. 2c. Tungsten was injected as a pellet into the LHD plasma. Numerous spectral lines from wide range of charge states of ions around $\mathrm{W}^{27+}$ in plasmas are overlapped and produce the UTA structure as measured. Table 1 shows the list of spectral lines labeled in Fig.2. Lines labeled a and b are blended. Our calculated wavelengths are 0.5 - $1.8 \AA$ shifted longer from data in NIST Atomic Spectra Database [14]. Lines b, c, and d are seen in the measured spectrum (Fig. 2c) and are shifted toward longer wavelength. Other peaks are from other charge states of tungsten ions (e.g., see Ref $[2])$.

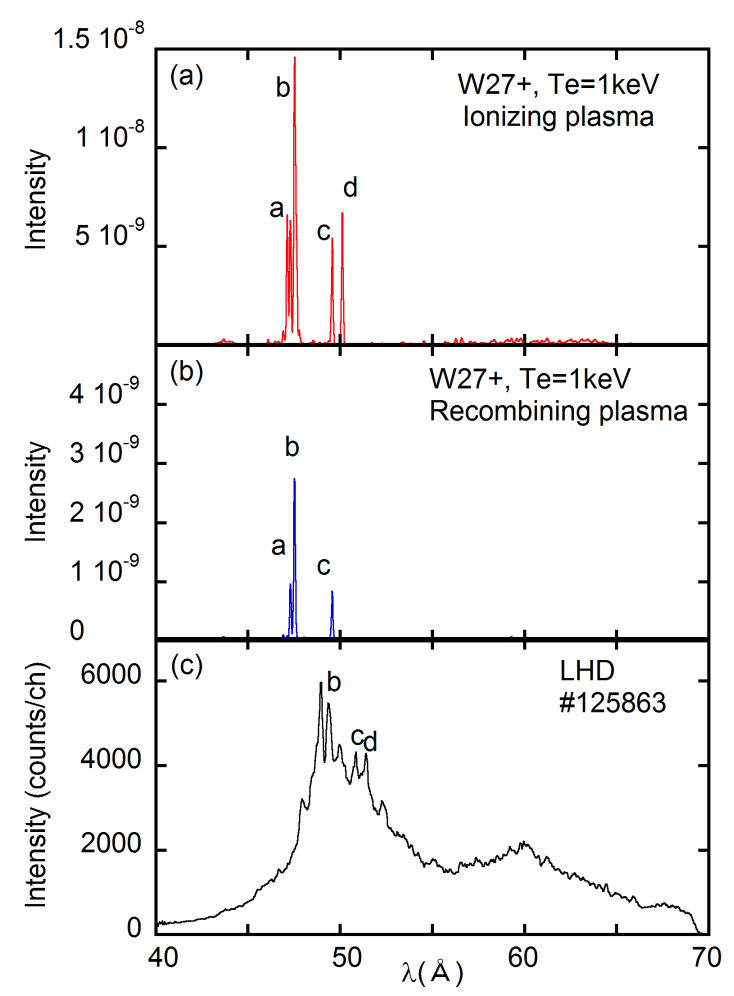

Fig. 2. EUV spectra of (a) ionizing plasma component and (b) recombining plasma component calculated for $\mathrm{T}_{e}=1 \mathrm{keV}$ and $\mathrm{n}_{e}=10^{13} \mathrm{~cm}^{-3}$. (c) Experimental tungsten UTA spectum measured in LHD plasma.

Interestingly, the strongest line is the same in both ionizing and recombining plasmas, but there are some differences. Spectral lines in blended a and b lines, and line $\mathrm{d}$ are weak in recombining plasma (see Table 1). Figures $3 \mathrm{a}$ and $3 \mathrm{~b}$ are close-up images of figure 2 to show weaker lines, with dielectronic satellite lines calculated from atomic data (Fig.3c). Details of the satellite line will be described in the next section. There are many weak lines at 55 - $66 \AA$ mainly due to $4 \mathrm{~d}$ - $4 \mathrm{f}$ transitions as described in Ref. [3].

As shown in Fig. 3a and 3b, these lines in this wavelength region in recombining plasma are all weak, similar 
Table 1. Spectral lines in Figure 2.

\begin{tabular}{cccccc}
\hline label & wavelengths $(\AA)$ & transition & $A_{r}\left(\mathrm{~s}^{-1}\right)^{\mathrm{a}}$ & note \\
& this work & $\mathrm{NIST}^{b}$ & & & \\
\hline a & 47.11 & 48.729 & $4 \mathrm{~d}^{10} 4 \mathrm{f}(\mathrm{J}=5 / 2)-4 \mathrm{~d}^{9} 4 \mathrm{f}^{2}(\mathrm{~J}=5 / 2)$ & $6.42(12)^{c}$ & weak in rec. \\
a & 47.28 & 48.729 & $4 \mathrm{~d}^{10} 4 \mathrm{f}(\mathrm{J}=7 / 2)-4 \mathrm{~d}^{9} 4 \mathrm{f}^{2}(\mathrm{~J}=7 / 2)$ & $6.98(12)$ & \\
b & 47.50 & 49.402 & $4 \mathrm{~d}^{10} 5 \mathrm{~s}(\mathrm{~J}=1 / 2)-4 \mathrm{~d}^{9} 4 \mathrm{f} 5 \mathrm{~s}(\mathrm{~J}=3 / 2)$ & $6.65(12)$ & \\
b & 47.54 & 49.402 & $4 \mathrm{~d}^{10} 4 \mathrm{f}(\mathrm{J}=7 / 2)-4 \mathrm{~d}^{9} 4 \mathrm{f}^{2}(\mathrm{~J}=5 / 2)$ & $5.94(12)$ & \\
b & 47.61 & 49.402 & $4 \mathrm{~d}^{10} 4 \mathrm{f}(\mathrm{J}=5 / 2)-4 \mathrm{~d}^{9} 4 \mathrm{f}^{2}(\mathrm{~J}=3 / 2)$ & $6.54(12)$ & weak in rec. \\
c & 49.55 & 50.895 & $4 \mathrm{~d}^{10} 4 \mathrm{f}(\mathrm{J}=7 / 2)-4 \mathrm{~d}^{9} 4 \mathrm{f}^{2}(\mathrm{~J}=9 / 2)$ & $4.12(12)$ & \\
d & 50.10 & 51.456 & $4 \mathrm{~d}^{10} 4 \mathrm{f}(\mathrm{J}=5 / 2)-4 \mathrm{~d}^{9} 4 \mathrm{f}^{2}(\mathrm{~J}=7 / 2)$ & $3.93(12)$ & weak in rec.
\end{tabular}

${ }^{a}$ Present calculations.

${ }^{b}$ NIST Atomic Spectra Data [14].

${ }^{c} \mathrm{x}(\mathrm{y})$ means $\mathrm{x} \times 10^{y}$.

to the lines in ionizing plasma. This means that the DR processes cannot contribute to the UTA peak at around 60 $\AA$ if the ion densities of $\mathrm{W}^{28+}$ and $\mathrm{W}^{27+}$ ions are about the same. However, this result does not deny the possibility suggested by Pütterich that the DR process may contribute to the UTA peak at around $60 \AA$, since tungsten ions in other charge states still have such a possibility. Similar studies to this case for other charge state ions are necessary.

\section{Dielectronic satellite lines and dielectronic}

\section{recombination rates}

DR satellite lines and DR rate coefficients can be estimated using the same atomic data in the $\mathrm{CR}$ model. DR satellite lines are the radiative transitions from the au- toionizing states to the bound states after the dielectronic capture to the autoionizing states.

The emission coefficient of the DR satellite line from the autoionizing state $i$ to the bound state $j$ after the dielectronic capture from the ionized ground state $i_{0}$ is obtained as

$$
\begin{aligned}
C_{S}^{e f f}\left(j, i, i_{0}\right)= & 3.3 \times 10^{-24}\left(\frac{I_{H}}{k T_{e}}\right)^{3 / 2} \frac{Q_{d}\left(j, i, i_{0}\right)}{g_{0}} \\
& \times \exp \left(-\frac{E_{s}(i)}{k T_{e}}\right)\left(\text { photonscm }^{3} \mathrm{~s}^{-1}\right),
\end{aligned}
$$

$$
Q_{d}\left(j, i, i_{0}\right)=\frac{g(i) A_{a}\left(i, i_{0}\right) A_{r}(i, j)}{\sum_{i_{0}^{\prime}} A_{a}\left(i, i_{0}^{\prime}\right)+\sum_{k} A_{r}(i, k)},
$$

where $I_{H}$ is the ionization potential of hydrogen, $k$ is the Boltzman constant, $T_{e}$ is the electron temperature, $g_{0}$ is the statistical weight of the ground state $i_{0}$ of $\mathrm{W}^{28+}$ ion, $E_{s}(i)$ is the energy difference between the autoionizing 


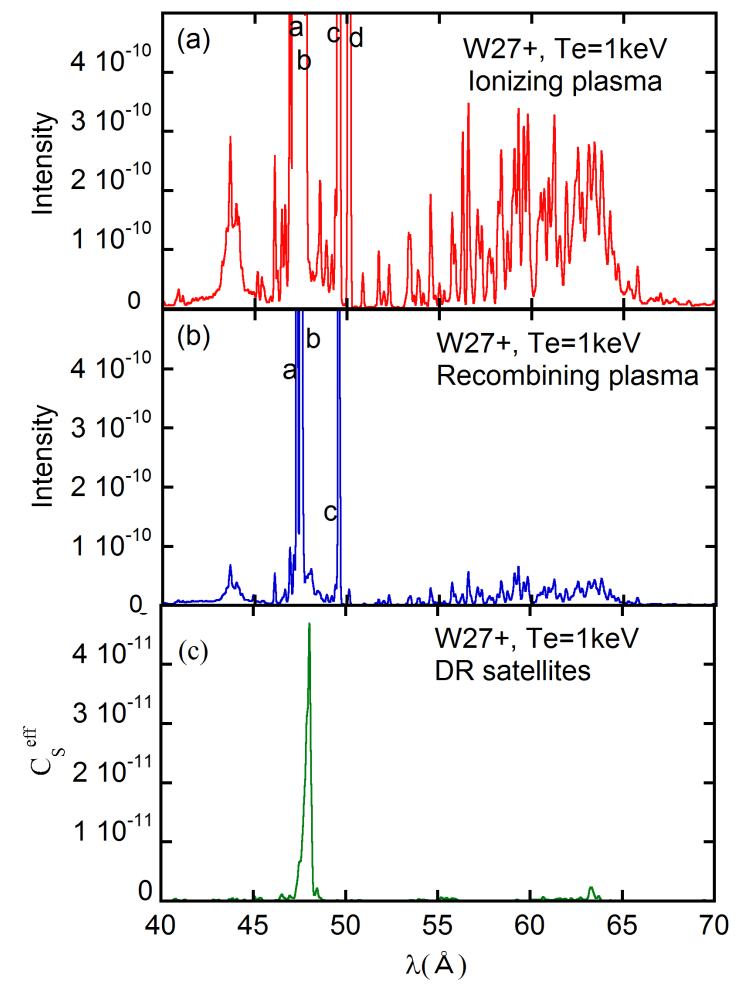

Fig. 3. (a) and (b) are the same as Fig.2 but with shorter vertical scale for weaker lines. (c) is effective emission coefficients of dielectronic satellite lines with $T_{e}=1 \mathrm{keV}$.

state $i$ and the ground state of $\mathrm{W}^{28+}$ ion, $Q_{d}$ is the intensity factor of the DR satellite line, and $g(i)$ is the statistical weight of the autoionizing state $i$. This intensity factor is determined by the branching ratio of the autoionizing states, and autoionizing rates to all possible channels to the ground state and excited states $i_{0}^{\prime}$ of $\mathrm{W}^{28+}$ ion for autoionization must be counted. We treat about 300 million transitions to obtain the intensity factors in eq.(5) for all satellite lines.

In addition, this is zero-density limit and any collisional processes which may change the population densities of the autoionizing states are not taken into account in

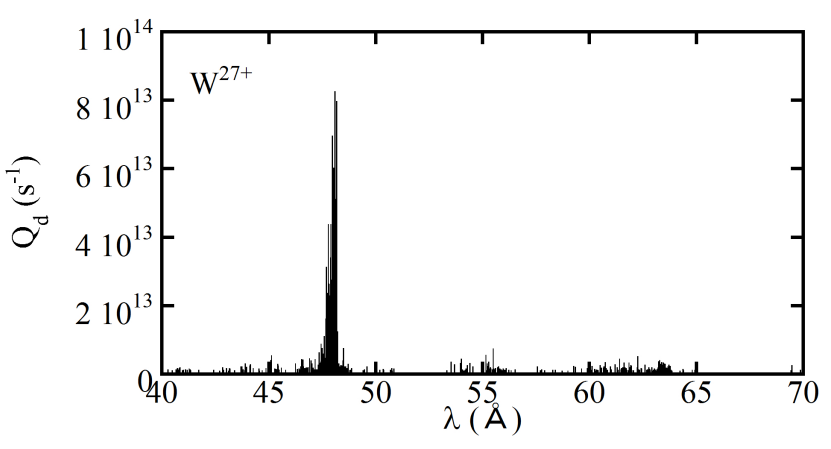

Fig. 4. Intensity factor $\mathrm{Q}_{d}$ (eq.(5)) at 40-70 $\AA$.

this formula. Acutual line intensities of the satellite lines in plasmas may be different from line intensitites estimated with eq.(4).

Figure 4 shows the intensity factor $Q_{d}$ at 40-70 $\AA$. A prominent satellite line due to many overlapping $4 \mathrm{~d}-4 \mathrm{f}$ transitions, such as $4 \mathrm{~d}^{10} 7 \mathrm{~g}-4 \mathrm{~d}^{9} 4 \mathrm{f} 7 \mathrm{~g}$, is found at $48 \AA$, and many weak lines at 55-65 $\AA$ region are seen. Figure 3c shows the emission coefficients of the DR satellite lines for electron temperature $1 \mathrm{keV}$, calculatd with eq.(4), and only one line at $48 \AA$ is strong. This satellite line is seen in the spectrum of recombining plasma (Fig.3b) and it is very weak compared with the resonance line labeled as b in Fig.2b.

Similarly, we can calculate the DR rate coefficient from the ground state of $\mathrm{W}^{28+}$ ion to $\mathrm{W}^{27+}$ ion as a function of electron temperature as

$$
\alpha_{d}^{t o t}\left(T_{e} ; i_{0}\right)=\sum_{i, j} C_{S}^{e f f}\left(j, i, i_{0} ; T_{e}\right),
$$

and the calculated rate is plotted in Fig. 5. In this calculation, all energy levels treated in the CR model are included, and we do not include any contributions from higher $n$ levels using $n$-scaling law as other works commonly did. 


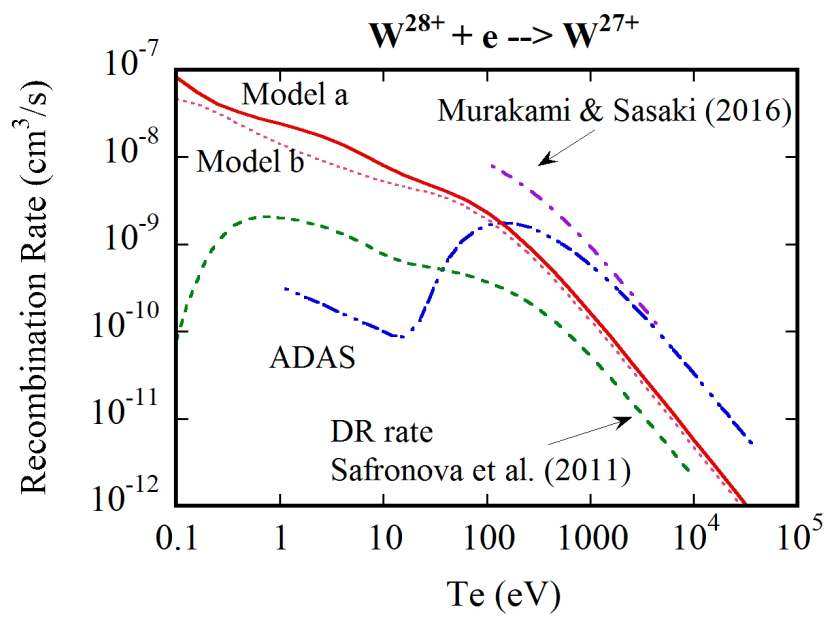

Fig. 5. Dielectronic recombination rate coefficients as a function of electron temperature. Model a (solid line) used the full set of the energy levels, and model b (dotted line) used the energy levels with $l \leq 4$. For comparison, DR rate obtained by Safronova [11], effecive recombination rates of ADAS [15] and Murakami and Sasaki [10] are plotted.

Model a in Fig, 5 is the DR rate coefficients calculated with the full set of the levels, and model b is calculated with levels with $l \leq 4$ to compare with the DR rate calculated by Safronova et al. [11] plotted in Fig.5. The difference between models a and $\mathrm{b}$ is about $20 \%$ at $T_{e}>100 \mathrm{eV}$ and becomes larger up to $90 \%$ at lower temperature.
In Fig. 5, We also plot effective recombination rates of ADAS ("acd5_w.dat") [15] and Murakami and Sasaki [10] for comparison.

The effective recombintion rate of ADAS is calculated by the $\mathrm{CR}$ model including radiative recombination rates and DR recombination rates. Those rates are originally taken from ADPACK datasets and DR rate are modified with a correction factor 1.52 estimated from the spectroscopic measurements in ASDEX Upgrade by Pütterich et al. [17].

ADPACK used an average ion model and DR rate was estimated with basically Burgess's empirical formula [18]. This formula is limited to moderate-charged low $\mathrm{Z}$ ions. Furthermore, the correction factor was estimated from the UTA intensity at $50 \AA$ by Pütterich et al. for $\mathrm{W}^{24+}$ - $\mathrm{W}^{35+}$ ions. So large ambiguity is included in the DR rate.

Nevertheless, the difference between this ADAS rate and our DR rate at $T_{e}>1 \mathrm{keV}$ is within factor $5-6$.

On the other hand, the effective recombination rate of Murakami and Sasaki [10] is obtained by the CA-CR model with large number of autoionizing states for limited

Safronova et al. considered autoionization states $4 \mathrm{~d}^{9} n^{\prime} l^{\prime} n l$ temperature range. In their model, the atomic structure with $n^{\prime} l^{\prime}=4$ f, $5 l$, and $6 l$, and $n \leq 9$ and $l \leq 4$, and higher $n$ contribution up to 1000 added using n-scaling law, which is about $20 \%$ contribution at $T_{e}>100 \mathrm{eV}$. Inclusion of $4 \mathrm{p}^{5} 4 \mathrm{~d}^{10} 4 \mathrm{f} n l$ levels $(n \leq 6, l \leq 5)$, which Safronova et al. did not include in their model, makes our DR rate coefficient higher than their model, especially at $T_{e}<100 \mathrm{eV}$. However, the difference at $T_{e}>1 \mathrm{keV}$ is within factor 3 . was determined with 50 core configurations plus outer $n l$ electron with $n$ up to 8 and $l$ up to 4 by using a computer algorithm [8]. Many autoionizing states allow many channels for dielectronic capture and following radiative decay, i.e., DR processes. Their effective rate is larger than our DR rate for factor 4-8. Note both rates do not include higher $n$ contributions. 
Thus it is found that different assumption on energy levels considered to calculate the DR rate makes differences between our DR rate and the rates of Safronova et al. and Murakami and Sasaki. Larger set of energy levels gives larger DR rate. Since Sasaki [8] examined the convergense on the recombination rates and other physical values obtained from the CA-CR model for larger set of energy levels, he found 50 core configurations gives results converged enough. Thus the rate of Murakami and Sasaki, obtained with the same method of Sasaki, can be thought as the upper limit for the DR rate. All the rates in Fig.5 agree within factor 6 at $T_{e}$ around $1 \mathrm{keV}$, with which $\mathrm{W}^{27+}$ and $\mathrm{W}^{28+}$ ions are usually observed in plasmas.

\section{Summary}

We have constructed the CR model for $\mathrm{W}^{27+}$ ions with 25,632 J-resolved fine structure levels, about $3 / 4$ of which are autoionizing levels. Further, we can calculate both ionizing and recombining plasma components of the population densities of the excited levels with this CR model, in which we considered electron-impact ionization, autoionization radiative recombination, three-body recombination, and dielectronic capture processes with radiative decay and electron-impact excitation and deexcitation processes. This is the first study on EUV spectra for recombining plasma and we obtained the EUV spectra of $\mathrm{W}^{27+}$ ion for both ionizing and recombining plasmas at $40-70 \AA$ region. The strongest line is the same in both ionizing and recombining plasmas, but some lines are much weaker in the recombining plasma than in the ionizing plasma. The many line features at $55-65 \AA$ are also weaker in the recombining plasma than in the ionizing plasma. Thus, in this ion case, DR process does not contribute to the second peak of UTA at $60 \AA$. We need to examine other ions to confirm the possibility of contribution of DR processes to the $60 \AA$ peak of the UTA.

Using the same atomic data with the CR model, we calculated the DR rate coefficient as a function of electron temperature. Compared with other rates obtained by other methods, our DR rate agree with other rates within factor 6 at $T_{e} \sim 1 \mathrm{keV}$. We will seek to extend our model for other charge states in future work.

This work was supported partly by KAKENHI (JSPS grantin-aid for scientific research) (B) 23340185, (A) 23246165 and (B) 16H04623. We acknowledge the LHD experiment group for their help on plasma experiments to measure tungsten spectra.

\section{References}

1. K. Asmussen, K. B. Fournier, J. M. Laming, R. Neu, J. F. Seely, R. Dux, W. Engelhardt, J. C. Fuchs, ASDEX Upgrade team, Nucl. Fusion 38, (1998) 967.

2. C. S. Harte, C. Suzuki, T. Kato, H. A. Sakaue, D. Kato, K. Sato, N. Tamura, S. Sudo, R. D'Arcy, E. Sokell, J. White, and G. O’Sullivan, J. Phys. B: At. Mol. Opt. Phys. 43, (2010) 205004 .

3. I. Murakami, H. A. Sakaue, C. Suzuki, D. Kato, M. Goto, N. Tamura, S. Sudo, S. Morita, LHD Experiment Group., Nucl. Fusion 55, (2015) 093016.

4. S. Morita, C. F. Dong, M. Goto et al., AIP Conf. Proc. 1545, (2013) 143. 
5. T. Pütterich, V. Jonauskas, R. Neu, R. Dux, ASDEX Upgrade Team, AIP Conf. Proc. 1545, (2013) 132.

6. M. Li et al, Plasma Sci. Tech. 16, (2014) 182.

7. S. P. Preval, N. R. Badnell, M. G. O’Mullane, Phys. Rev. A 93, (2016) 042703

8. A. Sasaki, I. Murakami, J. Phys. B: At. Mol. Opt. Phys. 46, (2013) 175701.

9. A. Sasaki, High Energy Density Phys. 9, (2013) 325.

10. I. Murakami, S. Sasaki, submitted to Proceedings of Workshop on Atomic and Molecular Processes in Plasma (Chengdu, China, July 26-28, 2016) (2016).

11. U. Safronova, A. S. Safronova, P. Beiersdorfer, W. R. Johnson, J. Phys. B: At. Mol. Opt. Phys. 44, (2011) 035005

12. B. W. Li, G. O’Sullivan, Y. B. Fu, C. Z. Dong, Phys. Rev. A 85, (2012) 052706.

13. A. Bar-Shalom, M. Klapisch and J. Oreg, J. Quant. Spectr. Radiant. Trans. 71, (2001) 169.

14. A. Kramida, Yu. Ralchenko, J. Reader, and NIST ASD Team (2015). NIST Atomic Spectra Database (ver. 5.3), [Online]. Available: http://physics.nist.gov/asd. National Institute of Standards and Technology, Gaithersburg, MD.

15. H. P. Summers, (2004) The ADAS User Manual, version 2.6 http://www.adas.ac.uk'

16. D. W. Post, R. V. Jensen, C. B. Tarter, W. H. Grasberger, W. A. Lokke, Atomic Data Nucl. Data Tables 20, (1977) 397.

17. T. Pütterich, R. Neu, R. Dux, A. D. Whiteford, M. G. O'Mullane and the ASDEX Upgrade Team, Plasma Phys. Control. Fusion 50, (2008) 085016.

18. A. Burgess, Astrophys. J. 141, (1965) 1588. 